\title{
The Relationship Between Adherence to the Mediterranean Diet and Body Composition in Nutrition and Dietetic Students
}

\section{Abstract}

Introduction: The determination of level of adherence to the Mediterranean diet (MD) in young adults has been investigated in countries situated near the Mediterranean region generally. The main purpose of this study were to determine differences in body composition by gender and level of adherence to the MD and to determine the relationship between body composition and level of adherence to the MD in Nutrition and Dietetics students at Near East University in Cyprus.

Materials and Methods: The study was conducted on 126 Nutrition and Dietetics students, aged 18 to 32 years. MDS was calculated, and then classified into three groups: good (36-55 points), moderate (21-35 points), and poor (0-20 points). Body composition was assessed using bioelectrical impedance (BIA). Anthropometrical measurements; height (H), body weight (BW), waist circumference (WC) and hip circumference (HC) were assessed according to standardized procedures and physical activity (PA) was assessed by using International Physical Activity Questionnaire (IPAQ-short form). The study protocol was approved by the Ethics Committee at Near East University.

Results: According to study results $31.0 \%$ of students were found out to low adherence, while $69.0 \%$ of students had moderate adherence to the MD. There is no student had high adherence to the MD. There was no significant difference $(p=0.877)$ between the male and female students in terms of adherence to Mediterranean diet. The findings indicate that the eating habits of the Nutrition and Dietetics students, even those studying nutrition, are in need of improvement. The results show that $78.6 \%$ of students were normal and $21.4 \%$ of students were overweight and obese. Male students' BW ( $p=0.000)$, WC $(p=0.000)$ and HC $(p=0.015)$ were higher than females', while female students' fat mass $(F M)(p=0.000)$ was higher than males'. However, there is no significant difference between BW $(p=0.724), F M(p=0.896)$, BMI $(p=0.691)$, WC $(p=0.632)$ and HC $(p=0.982)$ neither low adherence nor moderate adherence to the MD. Most of students minimal active according to IPAQ scores $(41.2 \%)$ and there is no significant difference IPAQ score and adherence to the MD $(\mathrm{p}=0.923)$.

Discussion: One of the healthiest diets worldwide is the traditional MD. Several studies have shown, that higher adherence to the MD is inversely related with BMI, waist circumference (WC) and waist-to-height ratio (WHR). Little is known of how adherence to the MD is related to body composition, especially in university students. Further large-scale studies are required to clarify the relationship between adherence to the MD and body composition

\section{Conflict of Interest}

There is no conflict of interest 